\title{
RESIDUES OF POLYGENIC FUNCTIONS*
}

BY

VINCENT C. POOR

1. Important results. A function $W(z)=u+i v$ is called polygenic if it is not analytic or monogenic. The delay in the study of such functions has doubtless been due to the fruitful field of analytic functions opened up by the uniqueness restriction on the derivative, $d w / d z$, of $w$. Some of the most important results in this relatively new field of polygenic functions are the areal derivative

$$
\frac{d w}{d \alpha}=\lim _{A \rightarrow 0} \frac{i \int_{c} w d z}{2 A}
$$

introduced with a different factor by Pompeiu $\dagger$ and

$$
\frac{d w}{d \beta}=\lim _{A \rightarrow 0} \frac{-\int_{c} w d \bar{z}}{2 i A}
$$

which in its essence is Kasner's $\ddagger$ mean derivative.

If $W$ is analytic in $z$ or $\bar{z}$, one of these circulation integrals is identically zero. Also from these two definitions the two circulation theorems§

$$
2 \int_{A} \frac{d w}{d \alpha} d A=-i \int_{c} w d z ; 2 i \int_{A} \frac{d w}{d \beta} d A=-\int_{c} w d \bar{z}
$$

are almost evident.

We define a polygenic function as regular at a point when it possesses a differential at that point. In the sequel we shall assume that the functions we consider are, in general, regular so that for a regular point, $a$, of a function $f(z)$ we will have

* Presented to the Society, November 30, 1929; received by the editors in September, 1929.

$\dagger$ Pompéiu, Sur une classe de fonctions d'une variable complexe, Rendiconti del Circolo Matematico di Palermo, vol. 23 (1st semester 1912), pp. 108-113.

$\ddagger$ Kasner, General theory of polygenic or non-monogenic functions. The derivative congruence of circles, Proceedings of the National Academy of Sciences, January, 1928, pp. 75-82. The notation here is due to G. Y. Rainich, University of Michigan. 12.

\& Calugaréano (Thesis), Sur les fonctions polygènes d'une variable complexe, Paris, 1928, pp. 11, 


$$
f(z)-f(a)=\frac{d f}{d \beta}(z-a)+\frac{d f}{d \alpha}(\bar{z}-\bar{a})+\eta
$$

where $d f / d \beta$ and $d f / d \alpha$ are the values of the derivatives at the point $a$, and $\eta$ is a quantity of higher order than the first in $z-a$, a quantity such that $\eta /(z-a)^{2}$ remains finite when $z$ goes to $a$.

In studying such functions we have as our model the highly developed theory of analytic or monogenic functions.

2. Zeros and poles. The simplest singularities of monogenic functions are poles which may be characterized as the zeros of the reciprocal of a given function. We begin therefore by a brief discussion of zeros of polygenic functions.

There is a large class of polygenic functions defined by a double power series*

$$
f(z)=a_{0}+a_{10} z+a_{01} \bar{z}+\cdots .
$$

Such a function vanishes at the origin if the absolute term, $a_{0}$, is taken equal to zero. In the case of a monogenic function the result of such vanishing is that a factor $z$ may be taken out so that the function can be represented in the forn

$$
f(z)=z F(z)
$$

where $F(z)$ is regular at the origin. A polygenic function of this form, where $F(z)$ is another polygenic function, also has a zero at the origin but this is by no means the only type of zeros. Without going far into the classification of zeros we consider in addition to this what seems to be the next simplest case, the case in which the whole linear term may be taken out as a factor so that

$$
f(z)=(a z+b \bar{z}) F(z)
$$

and we assume that $F(z)$ does not vanish in the neighborhood of the origin.

The function $a z+b \bar{z}$ evidently vanishes for $z=0$; to see whether it vanishes for other points in the neighborhood of the origin we notice that if $a z+b \bar{z}$ is zero we must have

or taking absolute values

$$
a z=-b \bar{z}
$$

$$
|a|=|b| \text {. }
$$

Thus if $|a| \neq|b|$ the origin is an isolated zero of $f(z)$. But if the relation

* Calugaréano, loc. cit., $\S 1$. 
$|a|=|b|$ or its equivalent $b=a e^{i \phi}$ holds we easily see that we have a whole line of zeros, distributed along the straight line whose slope angle is $(\phi-\pi) / 2$.

We pass to the consideration of poles which we define in accordance with the above, as points at which the function becomes infinite while its reciprocal is zero. Corresponding to the two simple types of zeros mentioned we consider two types of poles of $f(z)$ given by the equations

$$
f(z)=\frac{F(z)}{z} \text { and } f(z)=\frac{F(z)}{a z+b \bar{z}}
$$

respectively; here the pole is assumed, for convenience in writing, to be at the origin.

3. Residues. In the theory of monogenic functions the residue is defined by the equation

$$
R_{A}=\frac{1}{2 \pi i} \int_{c} f(z) d z
$$

If $f(z)$ has no poles inside the closed contour $c$, defining the region $A$, then $R_{A}$ is zero. If in this region $f(z)$ has a pole so that

$$
F(z)=(z-a) f(z),
$$

then

$$
R_{A}=F(a) .
$$

The only restriction on the contour $c$ is that the region $A$ bounded by it contains no other singularities of $f(z)$.

If, on the other hand, $f(z)$ is a polygenic function the contour integral is no longer independent of the path. Therefore the value of $R_{A}$ given above depends on the contour $c$, and the definition loses its sense. We may, however, define the residue of a polygenic function as the limit toward which the above expression tends when the contour $c$, assumed to be a circle* of radius $r$ with center at $a$, tends when the radius goes to zero. This definition may be regarded as a generalization of the above definition for monogenic functions since for such functions it furnishes the same value $R_{A}$.

Symbolically then we write

$$
R^{\prime}=\lim _{r \rightarrow 0} \frac{1}{2 \pi i} \int_{0} f(z) d z
$$

for our definition of the residue of a polygenic function at the point $z=a$ where the contour is a circle of radius $r$, with center at $a$.

* An arbitrary contour within the circle would serve the purpose just as well. 
TheOREM 1. If $f(z)$ is a polygenic function in a region $A$ and has no singularity at $z=a$ nor in the neighborhood of $z=a$ (and by this we mean that it is possible to construct a circle about the point a which contains no singularity of the function considered, except, possibly, at $z=a$ ) then

$$
R^{\prime}=0 \text {. }
$$

This, of course, is the analogue or generalization of Cauchy's first law.

We may write

$$
\int_{0} f(z) d z=\int_{0}[f(z)-f(a)] d z+f(a) \int_{0} d z .
$$

The last integral is evidently zero and the modulus of the preceding will be less than the maximum value of $|f(z)-f(a)|$ on the circle multiplied by $\int_{0}|d z|$. Both these factors tend towards zero with $r$ so that $R^{\prime}=0$.

Theorem 2. If $z=a$ is a simple isolated pole of the polygenic function $f(z)$, such that

$$
F(z)=(z-a) f(z)
$$

and $F(z)$ is regular at $a$, and in the neighborhood of $a$, then

$$
R^{\prime}=F(a) \text {. }
$$

This is the generalization of Cauchy's second law for monogenic functions.

We may write

$$
\int_{0} f(z) d z=\int_{0} \frac{F(z)-F(a)}{z-a} d z+F(a) \int_{0} \frac{d z}{z-a} .
$$

The second term in the right member is $2 \pi i F(a)$; the first term may be written according to the formula (1.1) as

$$
\frac{d f}{d \beta} \int_{0} d z+\frac{d f}{d \alpha} \int_{0} \frac{z-a}{\bar{z}-\bar{a}} d z+\int_{0} \frac{\eta}{z-a} d z .
$$

The first of these integrals vanishes and the other two tend towards zero with the radius of the circle, and the theorem is proved.

We consider now a more difficult problem of finding the residue at a pole of the second type of $\$ 2$. For convenience we shall take the singularity of the function $f(z)$ at the point $z=0$ and then study the behavior of the function at the origin. We assume then that

$$
(a z+b \bar{z}) f(z)=F(z)
$$

where $F(z)$ is regular in the neighborhood of $z=0$. In fact we will treat only the situation where $F(z)$ is unity so that 


$$
\int_{0} f(z) d z=\int_{0} \frac{d z}{a z+b \bar{z}} .
$$

We have to consider three different cases,

$$
|b|>|a| ;|b|<|a| ;|b|=|a| \text {. }
$$

We study together the first two cases.

Case I, $|b|>|a|$; Case II, $|b|<|a|$. If we change the form of the integral so that

$$
\int_{0} \frac{d z}{a z+b \bar{z}}=\frac{1}{2 a} \int_{0} \frac{2 a z d z}{a z^{2}+b r^{2}}
$$

we can easily evaluate it on the circle with a fixed radius $r$. Since in this form the denominator, if $r$ be regarded as fixed, is equal to an analytic function of $z$ and the numerator is its differential, we may apply the theorem in analytic function theory that such an integral is equal to the difference between the number of zeros and the number of poles of the function, in the area bounded by the contour of integration, multiplied by $2 \pi i$. In both cases considered, the function $a z^{2}+b r^{2}$ has no poles inside the circle of radius $r$.

In Case $I$ there are two zeros of the function outside the circle and no zeros inside; thus the integral vanishes in this case.

In Case II there are two zeros of the function $a z^{2}+b r^{2}$ inside the circle so that the integral is $2 \cdot 2 \pi i$. This argument holds for every $r$; we therefore have in the limit

$$
R^{\prime}=\lim _{r \rightarrow 0} \frac{1}{2 \pi i} \int_{0} f(z) d z=\frac{1}{a} .
$$

Case III, $|b|=|a|$. Here the poles of the function are distributed along a straight line through the origin. The integral in question is improper since the intersections of the line and the circle, radius $r$, make the integrand infinite. In this case $b=a e^{i \phi}$, where $(\phi-\pi) / 2$ is the slope angle of the line of poles, and the integral in (3.4) may be transformed as follows:

$$
\begin{aligned}
\int_{0} \frac{d z}{a z+b \bar{z}} & =\frac{1}{a} \int_{0} \frac{d z}{z+e^{i \phi \bar{z}}}=\frac{i}{a} \int_{0}^{2 \pi} \frac{e^{i \theta} d \theta}{e^{i \theta}+e^{i(\phi-\theta)}} \\
& =\frac{i}{a} \int_{0}^{2 \pi} \frac{d \theta}{1+e^{i(\phi-2 \theta)}}=\frac{i}{a} \int_{0}^{2 \pi} \frac{d \theta}{1+\cos (\phi-2 \theta)+i \sin (\phi-2 \theta)} \\
& =\frac{i}{a} \int_{0}^{2 \pi} \frac{[1+\cos (\phi-2 \theta)+i \sin (\phi-2 \theta)] d \theta}{2+2 \cos (\phi-2 \theta)} \\
& =\frac{i}{2 a} \int_{0}^{2 \pi} d \theta-\frac{1}{2 a} \int_{0}^{2 \pi} \tan \left(\theta-\frac{\phi}{2}\right) d \theta .
\end{aligned}
$$


Here the first integral evidently becomes $\pi i / a$. We agree to take the chief value of the second (improper) integral, letting $\theta$ go from $(\phi-\pi) / 2+\epsilon$ to $(\phi+\pi) / 2-\epsilon$ and from $(\phi+\pi) / 2+\epsilon$ to $(\phi+3 \pi) / 2-\epsilon$ and then making $\epsilon$ tend toward zero. If we pair off the elements of integration in the first and fourth quadrants and those in the second and third we see that we obtain zero.

If we think of $a z+b \bar{z}$ as a function of $\bar{z}$ and define the residue of its reciprocal in the same manner in $\bar{z}$ then $a r^{2}+b \bar{z}^{2}$ will be analytic in $\bar{z}$. We thus have a second residue

$$
\bar{R}=1 / b
$$

in Case I, zero in Case II, and $1 /(2 b)$ in Case III. If we define the total residue, $R$, as the sum of the residues in $z$ and $\bar{z}$ respectively, we will then have

Case I :

$$
\begin{aligned}
& R=1 / b ; \\
& R=1 / a ; \\
& R=(a+b) /(2 a b) .
\end{aligned}
$$

Case II :

Case III :

4. Areal derivative. An interesting application of this theory of residues arises in connection with the differentiation of

$$
\lim _{r \rightarrow 0} \int_{0} \frac{f(t) d t}{t-z}
$$

under the integral sign. According to Theorem 2 we may write equation (3.3)

$$
f(z)=\lim _{r \rightarrow 0} \frac{1}{2 \pi i} \int_{0} \frac{f(t) d t}{t-z}
$$

where the contour of integration is the circle of radius $r$, center at $z$. This is the generalization of Cauchy's integral representation of a function; in what follows we obtain the generalization for the integral representation of the derivative.

THEOREM 3. The $\beta$ derivative of a polygenic function may be expressed in the form

$$
\frac{d f}{d \beta}=\lim _{r \rightarrow 0} \frac{1}{2 \pi i} \int_{0} \frac{f(t) d t}{(t-z)^{2}}=\lim _{r \rightarrow 0} \frac{1}{2 \pi i} \int_{0} \frac{d}{d z} \frac{f(t)}{t-z} d t
$$

or the $\beta$ derivative may be obtained by taking the $z$ derivative in

under the integral sign.

$$
\lim _{r \rightarrow 0} \int_{0} \frac{f(t) d t}{t-z}
$$


If we use formula (1.1) we may write

$$
\begin{aligned}
\int_{0} \frac{f(t) d t}{(t-z)^{2}}=\int_{0} \frac{f(z) d t}{(t-z)^{2}}+\left(\frac{d f}{d \beta}\right)_{z} \int_{0} \frac{(t-z) d t}{(t-z)^{2}} & \\
& +\left(\frac{d f}{d \alpha}\right)_{z} \int_{0} \frac{\bar{t}-\bar{z}}{(t-z)^{2}} d t+\int_{0} \frac{\eta d t}{(t-z)^{2}} .
\end{aligned}
$$

To evaluate the right member we observe that the first integral falls under analytical function theory and that the integral vanishes identically. The last tends towards zero with $r$ since the integrand is finite. In the second and third integrals we make the substitution $r e^{i \theta}$ for $t-z$ and obtain

$$
\frac{d f}{d \beta} i \int_{0}^{2 \pi} d \theta+\frac{d f}{d \alpha} i \int_{0}^{2 \pi} \frac{\bar{t}-\bar{z}}{t-z} d \theta
$$

It will be seen that the second integral is identically zero; this leaves $2 \pi i(d f / d \beta)$ as our limit which proves the theorem.

The $\alpha$ derivative may be found in exactly the same way if we think of the polygenic function $f$ as a function of $\bar{z}$ and write

$$
f(\bar{z})=\lim _{r \rightarrow 0} \frac{1}{2 \pi i} \int_{0} \frac{-f(\bar{t}) d \bar{t}}{\bar{t}-\bar{z}} .
$$

This equation is the analogue to (3.3) and may be deduced in the same way.

Conclusion. In a new field it is frequently difficult to discriminate between the important and the unimportant. However, it would appear that the calculus of residues should play a very important rôle in certain phases of polygenic function theory.

UNIVERSITY OF MICHIGAN, ANn Arbor, Mich. 